\title{
CDH13 Polymorphisms are Associated with Adiponectin Levels and Metabolic Syndrome Traits Independently of Visceral Fat Mass
}

\author{
Aya Kitamoto ${ }^{1}$, Takuya Kitamoto ${ }^{1}$, Takahiro Nakamura², Tomoaki Matsuo ${ }^{3}$, Yoshio Nakata ${ }^{3}$, Hideyuki Hyogo ${ }^{4}$, \\ Hidenori Ochi ${ }^{5}$, Seika Kamohara ${ }^{6}$, Nobuyuki Miyatake ${ }^{7}$, Kazuaki Kotani ${ }^{8}$, Ikuo Mineo ${ }^{9}$, Jun Wada ${ }^{10}$, \\ Yuji Ogawa ${ }^{11}$, Masato Yoneda ${ }^{11}$, Atsushi Nakajima ${ }^{11}$, Tohru Funahashi ${ }^{12}$, Shigeru Miyazaki ${ }^{13}$, Katsuto Tokunaga ${ }^{14}$, \\ Hiroaki Masuzaki ${ }^{15}$, Takato Ueno ${ }^{16}$, Kazuaki Chayama ${ }^{5}$, Kazuyuki Hamaguchi ${ }^{17}$, Kentaro Yamada ${ }^{18}$, \\ Toshiaki Hanafusa $^{19}$, Shinichi Oikawa ${ }^{20}$, Toshiie Sakata ${ }^{21}$, Kiyoji Tanaka ${ }^{3}$, Yuji Matsuzawa ${ }^{8}$ and Kikuko Hotta ${ }^{22}$ \\ ${ }^{1}$ Pharmacogenomics, Kyoto University Graduate School of Medicine, Kyoto, Japan \\ ${ }^{2}$ Laboratory for Mathematics, National Defense Medical College, Tokorozawa, Japan \\ ${ }^{3}$ Graduate School of Comprehensive Human Sciences, University of Tsukuba, Tsukuba, Japan \\ ${ }^{4}$ Department of Gastroenterology and Hepatology, JA Hiroshima General Hospital, Hiroshima, Japan \\ ${ }^{5}$ Department of Gastroenterology and Metabolism, Institute of Biomedical and Health Sciences, Hiroshima University, Hiroshima, Japan \\ ${ }^{6}$ Health Science University, Yamanashi, Japan \\ ${ }^{7}$ Department of Hygiene, Faculty of Medicine, Kagawa University, Kagawa, Japan \\ ${ }^{8}$ Department of Metabolic Medicine, Graduate School of Medicine, Osaka University, Suita, Japan \\ ${ }^{9}$ Otemae Hospital, Osaka, Japan \\ ${ }^{10}$ Department of Medicine and Clinical Science, Okayama University Graduate School of Medicine, Dentistry and Pharmaceutical \\ Sciences, Okayama, Japan \\ ${ }^{11}$ Department of Gastroenterology and Hepatology, Yokohama City University School of Medicine, Yokohama, Japan \\ ${ }^{12}$ Department of Metabolism and Atherosclerosis, Graduate School of Medicine, Osaka University, Suita, Japan \\ ${ }^{13}$ Tokyo Postal Services Agency Hospital, Tokyo, Japan \\ ${ }^{14}$ Itami City Hospital, Itami, Japan \\ ${ }^{15}$ Division of Endocrinology, Diabetes and Metabolism, Hematology, Rheumatology, Graduate School of Medicine, University of \\ the Ryukyus, Okinawa, Japan \\ ${ }^{16}$ Research Center for Innovative Cancer Therapy, Kurume University, Kurume, Japan \\ ${ }^{17}$ Department of Practical Nursing Science, Faculty of Medicine, Oita University, Oita, Japan \\ ${ }^{18}$ Division of Endocrinology and Metabolism, Department of Medicine, Kurume University, Kurume, Japan \\ ${ }^{19}$ Department of Internal Medicine (I), Osaka Medical College, Takatsuki, Japan \\ ${ }^{20}$ Diabetes and Lifestyle Disease Center, Fukujuji Hospital, Tokyo, Japan \\ ${ }^{21}$ Department of Internal Medicine 1, Faculty of Medicine, Oita University, Oita, Japan \\ ${ }^{22}$ Department of Medical Innovation, Osaka University Hospital, Suita, Japan
}

Aim: Visceral fat accumulation contributes to the development of metabolic syndrome. As visceral fat accumulation increases, adiponectin levels decrease; therefore, adiponectin provides a link between visceral fat accumulation and metabolic disorders. Genome-wide association studies (GWASs) have identified genetic variations in the cadherin $13(\mathrm{CDH} 13)$ gene that are associated with adiponectin levels. Methods: We investigated whether single nucleotide polymorphisms (SNPs) in CDH13 was associated with adiponectin levels and metabolic syndrome traits independent of the visceral fat area (VFA), as measured using computed tomography (CT) in 945 Japanese individuals.

Results: We found that three CDH13 SNPs reported by recent GWASs (i.e., rs3865188, rs4783244, and rs12051272) were significantly associated with higher adiponectin levels $\left(P<1 \times 10^{-14}\right)$, even after adjustment for VFA. However, these adiponectin-inducing alleles of $C D H 13$ SNPs were significantly associated with traits consistent with deteriorating metabolic symptoms, such as higher fasting insulin, homeostasis model assessment-insulin resistance (HOMA-IR) scores, and triglycerides and lower highdensity lipoprotein (HDL)-cholesterol levels, similar to increasing VFA and decreasing adiponectin levels. Conclusion: These results suggested that $C D H 13$ SNPs cause an adiponectin-resistant status to compensate for increasing adiponectin levels and could result in the deterioration of metabolic syndrome traits.

\section{See editorial vol. 23: 292-294}

J Atheroscler Thromb, 2016; 23: 309-319.

Key words: $C D H 13$, Single nucleotide polymorphism, Adiponectin, Visceral fat area, Metabolic syndrome 


\section{Introduction}

Excess accumulation of adipose tissue, particularly visceral fat, contributes to the development of insulin resistance, resulting in symptoms characteristic of metabolic syndrome, including type 2 diabetes, dyslipidemia, and hypertension ${ }^{1,2)}$. In parallel with an increase in visceral fat, circulating adiponectin levels decrease, causing insulin resistance, type 2 diabetes, and cardiovascular disease, thereby providing a critical link between visceral fat accumulation and metabolic syndrome ${ }^{2-8)}$. However, in addition to visceral fat accumulation, genetic variations may affect adiponectin levels, as demonstrated in recent genome-wide association studies (GWASs) ${ }^{9-17)}$. One of the most important genetic variations determining adiponectin levels is located in the cadherin 13 (CDH13) gene, which encodes an adiponectin receptor ${ }^{18)}$.

Visceral fat is evaluated by measuring waist circumference, waist-hip ratio, bioelectrical impedance, or more precisely, the visceral fat area (VFA), determined by computed tomography $(\mathrm{CT})^{2)}$. Adiponectin levels are more strongly correlated with VFA than subcutaneous fat area $(\mathrm{SFA})^{8,19)}$. Although some studies have reported an association between polymorphisms in the $\mathrm{CDH} 13$ and metabolic syndrome traits ${ }^{20,21)}$, these studies did not measure VFA. Therefore, despite the potential link among VFA, adiponectin levels, and CDH13 polymorphisms, the effects of CDH13 genetic polymorphisms on circulating adiponectin levels and metabolic syndrome traits in individuals with varying VFAs remain unclear.

\section{Aim}

We investigated whether the effects of $\mathrm{CDH} 13$ polymorphisms on adiponectin levels and metabolic syndrome traits are dependent on VFA.

\section{Methods}

\section{Participants}

Japanese participants $(n=945)$ were recruited for this study. Patients with a disease (such as cancer, renal disease, cardiovascular disease, and hepatic failure) or receiving medication (i.e., insulin, thiazolidinedione, corticosteroids, or chemotherapy) that would be

Address for correspondence: Kikuko Hotta, Department of Medical Innovation, Osaka University Hospital, 2-2

Yamadaoka, Suita, Osaka 565-0871, Japan

E-mail: hottakikuko@dmi.med.osaka-u.ac.jp

Received: June 7, 2015

Accepted for publication: August 16, 2015 expected to affect body weight or adiponectin levels were excluded. The participants agreed to undergo CT to determine VFA and SFA at the umbilical level (L4L5), as previously reported ${ }^{22)}$. VFA and SFA were calculated using the FatScan software program (N2system, Osaka, Japan) ${ }^{22)}$. Clinical data, which were recorded at the first visit to the hospital, are summarized in Table 1. Homeostasis model assessment-insulin resistance (HOMA-IR) scores were assessed as fasting insulin $(\mu \mathrm{U} / \mathrm{mL}) \times$ fasting plasma glucose $(\mathrm{mg} /$ $\mathrm{dL}) / 405$. The recruitment and study protocols were conducted in compliance with the Declaration of Helsinki, and all participants provided written informed consent. The study was approved by Kyoto University (G374).

\section{DNA Extraction and Single Nucleotide Polymorphism (SNP) Genotyping}

Genomic DNA was extracted from blood samples that were collected from each participant using a Genomix kit (Talent Srl, Trieste, Italy). We selected three SNPs (rs3865188, rs4783244, and rs12051272) in the $C D H 13$ gene that were recently identified by GWASs, 12-15) and constructed Invader probes (Third Wave Technologies, Madison, WI, USA) for these SNPs. The SNPs were genotyped using Invader assays, as previously described ${ }^{23)}$, and used for analysis. The success rate of these assays was $>99.7 \%$.

\section{Measurement of Plasma Adiponectin Levels}

Plasma total adiponectin levels were measured using an enzyme-linked immunosorbent assay (ELISA; human adiponectin ELISA kit; Otsuka Pharmaceutical Co., Tokushima, Japan).

\section{Statistical Analysis}

For the additive model, we characterized the genotypes as 0,1 , or 2, depending on the number of copies of the tested alleles. Tested alleles were defined as previously identified adiponectin-inducing alleles ${ }^{9,12-15)}$. Multiple linear regression analyses were performed to test the independent effects of each allele from each SNP on adiponectin and metabolic syndrome traits, accounting for the effects of other variables [i.e., age, sex, body mass index (BMI), VFA, and SFA]. Values for BMI, VFA, SFA, fasting glucose, fasting insulin, HOMA-IR, triglycerides, and adiponectin were logarithmically transformed before performing multiple linear regression analysis. Linkage disequilibrium was calculated using Haploview ${ }^{24)}$.

A structural equation modeling (SEM) analysis was used to assess the contribution of CDH13 SNPs to the circulating adiponectin levels and metabolic 
Table 1. Clinical characteristics of the participants

\begin{tabular}{lccc}
\hline & Men & Women & Total \\
\hline$n$ & 412 & 533 & 945 \\
\hline Age $($ year $)$ & $50.6 \pm 11.1$ & $51.8 \pm 10.4$ & $51.3 \pm 10.7$ \\
BMI $\left(\mathrm{kg} / \mathrm{m}^{2}\right)$ & $29.5 \pm 5.0$ & $27.3 \pm 4.4$ & $28.2 \pm 4.8$ \\
VFA $\left(\mathrm{cm}^{2}\right)$ & $161.5 \pm 66.9$ & $93.6 \pm 50.1$ & $123.2 \pm 67.0$ \\
SFA $\left(\mathrm{cm}^{2}\right)$ & $202.3 \pm 98.5$ & $239.0 \pm 89.0$ & $223.0 \pm 94.9$ \\
V/S $\left(\mathrm{cm}^{2}\right)$ & $0.9 \pm 0.4$ & $0.4 \pm 0.2$ & $0.6 \pm 0.4$ \\
Fasting plasma glucose $(\mathrm{mg} / \mathrm{dL})$ & $103.9 \pm 29.0$ & $97.5 \pm 20.7$ & $100.3 \pm 24.9$ \\
Insulin $(\mu \mathrm{U} / \mathrm{mL})$ & $11.7 \pm 14.8$ & $8.9 \pm 8.1$ & $10.1 \pm 11.5$ \\
HOMA-IR & $3.2 \pm 4.7$ & $2.2 \pm 2.2$ & $2.6 \pm 3.6$ \\
Total cholesterol (mg/dL) & $213.3 \pm 36.8$ & $221.8 \pm 36.6$ & $218.1 \pm 36.9$ \\
Triglycerides $(\mathrm{mg} / \mathrm{dL})$ & $164.1 \pm 158.4$ & $108.1 \pm 76.2$ & $132.5 \pm 122.3$ \\
HDL-cholesterol $(\mathrm{mg} / \mathrm{dL})$ & $53.2 \pm 14.0$ & $62.7 \pm 15.5$ & $58.6 \pm 15.6$ \\
Systolic blood pressure $(\mathrm{mmHg})$ & $132.4 \pm 17.0$ & $130.5 \pm 19.0$ & $131.3 \pm 18.2$ \\
Diastolic blood pressure $(\mathrm{mmHg})$ & $86.3 \pm 12.3$ & $81.1 \pm 10.8$ & $83.4 \pm 11.7$ \\
Adiponectin $(\mu \mathrm{g} / \mathrm{mL})$ & $6.1 \pm 3.1$ & $9.8 \pm 5.0$ & $8.2 \pm 4.7$ \\
\hline
\end{tabular}

HOMA-IR was assessed as fasting insulin $(\mu \mathrm{U} / \mathrm{mL}) \times$ fasting plasma glucose $(\mathrm{mg} / \mathrm{dL}) / 405$.

syndrome, as described in a previous report ${ }^{25}$, with some modifications. The path diagram is shown in Fig. 1. The CDH13 rs12051272 genotype, visceral fat accumulation, and decreased adiponectin levels were hypothesized to be upstream of the clustering of metabolic abnormalities (risk clustering). Risk clustering was assumed to be measured by fasting glucose, HOMA-IR, triglycerides, high-density lipoprotein (HDL)-cholesterol, and systolic and diastolic blood pressures. The effects of age and sex on VFA and adiponectin levels were added in this path diagram.

All statistical analyses were performed using $\mathrm{R}$ software (http://www.r-project.org/). SEM analysis was performed using the lavaan package in $\mathrm{R}^{26)}$. Differences with $P$ values of $<0.016$ (0.05/3 SNPs) were considered statistically significant.

\section{Results}

\section{Effects of SNPs, BMI, VFA, and SFA on Adiponectin Levels}

The clinical characteristics and genotypes of the participants are shown in Tables $\mathbf{1}$ and 2. All SNPs were in Hardy-Weinberg equilibrium $(P>0.5)$. The frequencies of adiponectin-inducing alleles did not diverge from those reported in the HapMap database. As previously reported, adiponectin-inducing alleles of the three SNPs (rs3865188, rs4783244, and rs12051272) were associated with higher adiponectin levels (Table 2). Multiple linear regression analyses indicated that these associations were significant after adjustment for age, sex, and anthropometric parameters (BMI, VFA, and SFA; Table 3). Among the anthropometric parameters, VFA was most strongly associated with adiponectin levels, even after adjustment for BMI. Each adiponectin-inducing allele of the three SNPs showed stronger associations with adiponectin levels than BMI and SFA $\left(P<1 \times 10^{-14}\right)$. Therefore, we used VFA, instead of BMI, as an explanatory variable in the following analyses of allelic effects on metabolic syndrome traits.

\section{Effects of SNPs, VFA, and Adiponectin Levels on Metabolic Syndrome Traits}

Further, we examined the allelic effect of SNPs on metabolic syndrome traits (Table 4). Higher VFA and lower adiponectin levels were significantly associated with higher fasting plasma glucose, insulin, HOMA-IR, and triglycerides and lower HDL-cholesterol levels, confirming the results of several previous reports $^{3,6-8)}$. Although adiponectin-inducing alleles of the three SNPs were associated with higher adiponectin levels, these alleles were significantly associated with higher fasting insulin, HOMA-IR, and triglycerides and lower HDL-cholesterol levels. The effect of these SNPs (i.e., promoting deterioration of metabolic syndrome traits) was unrelated to higher adiponectin and lower VFA levels. Thus, the allelic effects of the three SNPs on adiponectin and metabolic syndrome traits were contradictory. The adiponectin-inducing alleles caused deterioration of metabolic syndrome traits, confirming the results of a previous report ${ }^{21)}$. 




Fig. 1. Effects of the CDH13 rs12051272 genotype, adiponectin levels, and VFA on metabolic syndrome

Risk clustering was a latent variable measured by plasma glucose (PG), HOMA-IR, triglycerides (TG), HDL-cholesterol (HDL), and systolic (SBP) and diastolic (DBP) blood pressures. Rectangles are observed variables, and $\mathrm{e}_{1}$ to $\mathrm{e}_{9}$ are error variables. Each value on the arrow indicates the standardized regression weight.

Table 2. Genotypic characteristics of participants

\begin{tabular}{|c|c|c|c|c|c|c|c|c|c|}
\hline \multirow{2}{*}{$\begin{array}{l}\text { SNP } \\
\text { Genotype }\end{array}$} & \multicolumn{3}{|c|}{ rs3865188 } & \multicolumn{3}{|c|}{ rs 4783244} & \multicolumn{3}{|c|}{ rs 12051272} \\
\hline & $\mathrm{AA}^{*}$ & AT & TT & $\mathrm{CC}^{*}$ & $\mathrm{CA}$ & $\mathrm{AA}$ & $\mathrm{GG}^{*}$ & GT & $\mathrm{TT}$ \\
\hline$n$ & 449 & 397 & 97 & 451 & 400 & 92 & 452 & 402 & 91 \\
\hline Age (year) & $51.1 \pm 10.9$ & $51.4 \pm 10.8$ & $51.4 \pm 9.5$ & $51.0 \pm 10.9$ & $51.5 \pm 10.6$ & $51.5 \pm 9.9$ & $51.0 \pm 10.9$ & $51.5 \pm 10.7$ & $51.7 \pm 9.7$ \\
\hline BMI $\left(\mathrm{kg} / \mathrm{m}^{2}\right)$ & $28.3 \pm 4.9$ & $28.1 \pm 4.8$ & $28.3 \pm 4.2$ & $28.4 \pm 5.0$ & $28.1 \pm 4.7$ & $28.2 \pm 4.3$ & $28.4 \pm 5.0$ & $28.1 \pm 4.7$ & $28.3 \pm 4.2$ \\
\hline VFA $\left(\mathrm{cm}^{2}\right)$ & $122.6 \pm 69.1$ & $124.8 \pm 67.4$ & $120.2 \pm 55.9$ & $123.7 \pm 69.4$ & $123.2 \pm 66.9$ & $120.9 \pm 56.1$ & $123.7 \pm 69.7$ & $123.1 \pm 66.4$ & $121.2 \pm 56.4$ \\
\hline $\mathrm{SFA}\left(\mathrm{cm}^{2}\right)$ & $228.1 \pm 98.1$ & $215.8 \pm 89.1$ & $228.2 \pm 102.6$ & $228.5 \pm 98.2$ & $216.7 \pm 89$ & $222.8 \pm 103.8$ & $228.7 \pm 98.4$ & $216.1 \pm 89.0$ & $225.8 \pm 102.2$ \\
\hline Plasma glucose $(\mathrm{mg} / \mathrm{dL})$ & $100 \pm 22.5$ & $100.6 \pm 24.3$ & $100.3 \pm 35.6$ & $100 \pm 22.4$ & $100.5 \pm 24.3$ & $100.7 \pm 36.5$ & $100 \pm 22.4$ & $100.5 \pm 24.2$ & $101.2 \pm 36.6$ \\
\hline Insulin $(\mu \mathrm{U} / \mathrm{mL})$ & $10.9 \pm 12.7$ & $9.7 \pm 11.1$ & $8.7 \pm 6.5$ & $10.9 \pm 12.8$ & $9.5 \pm 11$ & $8.9 \pm 6.6$ & $10.9 \pm 12.8$ & $9.5 \pm 10.9$ & $8.9 \pm 6.6$ \\
\hline HOMA-IR & $2.8 \pm 3.8$ & $2.5 \pm 3.5$ & $2.2 \pm 2.1$ & $2.8 \pm 3.9$ & $2.5 \pm 3.5$ & $2.3 \pm 2.2$ & $2.8 \pm 3.8$ & $2.5 \pm 3.5$ & $2.3 \pm 2.2$ \\
\hline Total cholesterol (mg/dL) & $217.5 \pm 36.1$ & $219.6 \pm 37.9$ & $214.7 \pm 36.9$ & $217.4 \pm 36.2$ & $219.9 \pm 37.7$ & $214 \pm 37.4$ & $217.6 \pm 36.1$ & $219.4 \pm 37.8$ & $214.6 \pm 37.3$ \\
\hline Triglycerides (mg/dL) & $131.7 \pm 92.4$ & $136.1 \pm 157.8$ & $122.5 \pm 66$ & $130.7 \pm 92$ & $136.6 \pm 157.5$ & $123.6 \pm 67.1$ & $130.7 \pm 92.0$ & $136.6 \pm 157$ & $123.5 \pm 67.1$ \\
\hline HDL-cholesterol (mg/dL) & $58.0 \pm 15.3$ & $58.9 \pm 15.7$ & $59.3 \pm 16.1$ & $58.2 \pm 15.3$ & $58.8 \pm 15.6$ & $59.2 \pm 16.6$ & $58.2 \pm 15.4$ & $58.9 \pm 15.6$ & $58.9 \pm 16.5$ \\
\hline Systolic blood pressure (mmHg) & $130.9 \pm 18.1$ & $131.4 \pm 17.6$ & $133 \pm 20.9$ & $131 \pm 18.3$ & $131.2 \pm 17.4$ & $133.2 \pm 20.8$ & $131.0 \pm 18.2$ & $131.3 \pm 17.5$ & $133.3 \pm 20.8$ \\
\hline Diastolic blood pressure $(\mathrm{mmHg})$ & $83.5 \pm 11.8$ & $83.2 \pm 11.6$ & $83.3 \pm 11.6$ & $83.6 \pm 11.9$ & $83.2 \pm 11.6$ & $83.1 \pm 11.3$ & $83.6 \pm 11.9$ & $83.2 \pm 11.6$ & $83.1 \pm 11.3$ \\
\hline Adiponectin $(\mu \mathrm{g} / \mathrm{mL})$ & $9.1 \pm 5.1$ & $7.5 \pm 4.1$ & $6.4 \pm 3.5$ & $9.1 \pm 5.1$ & $7.6 \pm 4.2$ & $6.3 \pm 3.4$ & $9.1 \pm 5.1$ & $7.5 \pm 4.1$ & $6.2 \pm 3.5$ \\
\hline
\end{tabular}

\footnotetext{
*adiponectin-inducing allele.
} 
Table 3. Effects of SNPs, BMI, VFA, and SFA on plasma adiponectin levels

\begin{tabular}{|c|c|c|c|c|c|c|}
\hline \multirow[b]{2}{*}{ Model 1} & \multicolumn{2}{|c|}{ rs3865188 } & \multicolumn{2}{|c|}{ rs 4783244} & \multicolumn{2}{|c|}{ rs12051272 } \\
\hline & $\beta$ (s.e.) & $P$ & $\beta$ (s.e.) & $P$ & $\beta$ (s.e.) & $P$ \\
\hline SNP & $0.075(0.009)$ & $3.6 \times 10^{-15}$ & $0.078(0.009)$ & $2.0 \times 10^{-16}$ & $0.082(0.009)$ & $1.5 \times 10^{-17}$ \\
\hline Age & $0.004(0.001)$ & $1.5 \times 10^{-13}$ & $0.004(0.001)$ & $8.8 \times 10^{-14}$ & $0.004(0.001)$ & $5.7 \times 10^{-14}$ \\
\hline Sex & $-0.183(0.013)$ & $4.8 \times 10^{-42}$ & $-0.184(0.013)$ & $1.9 \times 10^{-42}$ & $-0.183(0.013)$ & $1.5 \times 10^{-42}$ \\
\hline $\log _{10}(\mathrm{BMI})$ & $-0.317(0.092)$ & $6.2 \times 10^{-4}$ & $-0.323(0.092)$ & $4.7 \times 10^{-4}$ & $-0.321(0.092)$ & $4.8 \times 10^{-4}$ \\
\hline Model 2 & $\beta$ (s.e.) & $P$ & $\beta$ (s.e.) & $P$ & $\beta$ (s.e.) & $P$ \\
\hline SNP & $0.074(0.009)$ & $7.8 \times 10^{-16}$ & $0.079(0.009)$ & $1.3 \times 10^{-17}$ & $0.081(0.009)$ & $9.5 \times 10^{-19}$ \\
\hline Age & $0.006(0.001)$ & $1.0 \times 10^{-21}$ & $0.006(0.001)$ & $3.1 \times 10^{-22}$ & $0.006(0.001)$ & $1.8 \times 10^{-22}$ \\
\hline Sex & $-0.131(0.014)$ & $1.1 \times 10^{-20}$ & $-0.131(0.014)$ & $1.0 \times 10^{-20}$ & $-0.131(0.014)$ & $8.4 \times 10^{-21}$ \\
\hline $\log _{10}(\mathrm{VFA})$ & $-0.233(0.025)$ & $8.1 \times 10^{-20}$ & $-0.236(0.025)$ & $2.1 \times 10^{-20}$ & $-0.236(0.025)$ & $1.8 \times 10^{-20}$ \\
\hline Model 3 & $\beta$ (s.e.) & $P$ & $\beta$ (s.e.) & $P$ & $\beta$ (s.e.) & $P$ \\
\hline SNP & $0.073(0.009)$ & $1.0 \times 10^{-15}$ & $0.078(0.009)$ & $1.7 \times 10^{-17}$ & $0.081(0.009)$ & $1.1 \times 10^{-18}$ \\
\hline Age & $0.006(0.001)$ & $4.4 \times 10^{-23}$ & $0.006(0.001)$ & $1.4 \times 10^{-23}$ & $0.006(0.001)$ & $7.4 \times 10^{-24}$ \\
\hline Sex & $-0.128(0.014)$ & $1.4 \times 10^{-19}$ & $-0.127(0.014)$ & $1.2 \times 10^{-19}$ & $-0.127(0.014)$ & $1.1 \times 10^{-19}$ \\
\hline $\log _{10}(\mathrm{VFA})$ & $-0.282(0.031)$ & $1.1 \times 10^{-18}$ & $-0.284(0.031)$ & $3.7 \times 10^{-19}$ & $-0.284(0.031)$ & $3.0 \times 10^{-19}$ \\
\hline $\log _{10}(\mathrm{BMI})$ & $0.286(0.111)$ & 0.010 & $0.284(0.11)$ & 0.010 & $0.286(0.11)$ & 0.0094 \\
\hline Model 4 & $\beta$ (s.e.) & $P$ & $\beta$ (s.e.) & $P$ & $\beta$ (s.e.) & $P$ \\
\hline SNP & $0.075(0.009)$ & $3.7 \times 10^{-15}$ & $0.079(0.009)$ & $2.0 \times 10^{-16}$ & $0.082(0.009)$ & $1.5 \times 10^{-17}$ \\
\hline Age & $0.004(0.001)$ & $5.6 \times 10^{-14}$ & $0.005(0.001)$ & $3.5 \times 10^{-14}$ & $0.005(0.001)$ & $2.1 \times 10^{-14}$ \\
\hline Sex & $-0.202(0.013)$ & $2.7 \times 10^{-49}$ & $-0.203(0.013)$ & $5.0 \times 10^{-50}$ & $-0.202(0.013)$ & $5.1 \times 10^{-50}$ \\
\hline $\log _{10}(\mathrm{SFA})$ & $-0.090(0.034)$ & 0.0087 & $-0.094(0.034)$ & 0.0060 & $-0.092(0.034)$ & 0.0068 \\
\hline Model 5 & $\beta$ (s.e.) & $P$ & $\beta$ (s.e.) & $P$ & $\beta$ (s.e.) & $P$ \\
\hline SNP & $0.075(0.009)$ & $3.8 \times 10^{-15}$ & $0.078(0.009)$ & $2.2 \times 10^{-16}$ & $0.081(0.009)$ & $1.6 \times 10^{-17}$ \\
\hline Age & $0.004(0.001)$ & $1.6 \times 10^{-13}$ & $0.004(0.001)$ & $9.8 \times 10^{-14}$ & $0.004(0.001)$ & $6.1 \times 10^{-14}$ \\
\hline Sex & $-0.182(0.016)$ & $1.1 \times 10^{-29}$ & $-0.184(0.016)$ & $3.3 \times 10^{-30}$ & $-0.183(0.015)$ & $3.8 \times 10^{-30}$ \\
\hline $\log _{10}(\mathrm{SFA})$ & $0.005(0.055)$ & 0.93 & $-0.001(0.055)$ & 0.98 & $0.002(0.055)$ & 0.97 \\
\hline $\log _{10}(\mathrm{BMI})$ & $-0.326(0.148)$ & 0.028 & $-0.32(0.148)$ & 0.031 & $-0.326(0.147)$ & 0.027 \\
\hline
\end{tabular}

The effect sizes and $P$-values were derived from linear regression analysis. Adiponectin was log-transformed for the analyses.

\section{SEM Analysis of the Effects of SNPs on Adiponectin Levels and Metabolic Syndrome}

In order to assess the effects of $C D H 13$ SNPs on adiponectin levels and metabolic syndrome, we performed SEM analysis. Fig. 1 shows the results of the SNP rs12051272, which had the best genotyping success rate. $P$-values of all estimates in the path diagram were less than 0.005 . The path diagram comparative fit index (CFI) was 0.634 , and the root mean square error of approximation was 0.18 . The rs12051272 genotype significantly increased adiponectin levels to be protective against metabolic syndrome (Fig. 1). On the other hand, the rs12051272 genotype significantly increased the risk clustering, prompting metabolic syndrome. SEM analysis using other SNPs (rs3865188 and rs4783244) showed similar results (data not shown).

\section{Discussion}

Adiponectin is specifically secreted from adipose tissue, and its plasma levels are decreased during visceral fat accumulation ${ }^{2-8,19)}$.

In this study, we found that adiponectin levels were determined by $\mathrm{CDH} 13$ polymorphisms, independent of visceral fat accumulation and subcutaneous and total fat mass. This is the first report demonstrating that the allelic effects of $\mathrm{CDH} 13$ polymorphisms on adiponectin levels were as strong as those of visceral fat accumulation. Thus, our data suggested that the clinical study of the metabolic syndrome 
Table 4. Effects of SNP, age, sex, VFA, and adiponectin levels on metabolic syndrome traits

\begin{tabular}{|c|c|c|c|c|c|c|}
\hline \multirow[t]{2}{*}{ SNP ID } & \multicolumn{2}{|c|}{ rs3865188 } & \multicolumn{2}{|c|}{ rs4783244 } & \multicolumn{2}{|c|}{ rs12051272 } \\
\hline & $\beta$ (s.e.) & $P$ & $\beta$ (s.e.) & $P$ & $\beta$ (s.e.) & $P$ \\
\hline \multicolumn{7}{|l|}{ Plasma glucose } \\
\hline SNP & $0.005(0.004)$ & 0.23 & $0.005(0.004)$ & 0.23 & $0.004(0.004)$ & 0.29 \\
\hline Age & $0.001(0.000)$ & $4.7 \times 10^{-5}$ & $0.001(0.000)$ & $4.2 \times 10^{-5}$ & $0.001(0.000)$ & $4.6 \times 10^{-5}$ \\
\hline Sex & $-0.002(0.006)$ & 0.73 & $-0.002(0.006)$ & 0.74 & $-0.002(0.006)$ & 0.70 \\
\hline $\log _{10}(\mathrm{VFA})$ & $0.068(0.011)$ & $1.4 \times 10^{-9}$ & $0.067(0.011)$ & $2.0 \times 10^{-9}$ & $0.067(0.011)$ & $1.6 \times 10^{-9}$ \\
\hline $\log _{10}$ (adiponectin) & $-0.047(0.014)$ & $6.8 \times 10^{-4}$ & $-0.047(0.014)$ & $6.6 \times 10^{-4}$ & $-0.047(0.014)$ & $6.8 \times 10^{-4}$ \\
\hline \multicolumn{7}{|l|}{ Insulin } \\
\hline SNP & $0.048(0.015)$ & 0.0012 & $0.045(0.015)$ & 0.0026 & $0.046(0.015)$ & 0.0020 \\
\hline Age & $-0.004(0.001)$ & $7.7 \times 10^{-6}$ & $-0.004(0.001)$ & $8.9 \times 10^{-6}$ & $-0.004(0.001)$ & $1.0 \times 10^{-5}$ \\
\hline Sex & $-0.071(0.023)$ & 0.0019 & $-0.070(0.023)$ & 0.0020 & $-0.071(0.023)$ & 0.0019 \\
\hline $\log _{10}(\mathrm{VFA})$ & $0.435(0.041)$ & $1.4 \times 10^{-24}$ & $0.433(0.041)$ & $2.8 \times 10^{-24}$ & $0.433(0.041)$ & $2.5 \times 10^{-24}$ \\
\hline $\log _{10}$ (adiponectin) & $-0.233(0.052)$ & $6.9 \times 10^{-6}$ & $-0.233(0.052)$ & $7.8 \times 10^{-6}$ & $-0.236(0.052)$ & $6.2 \times 10^{-6}$ \\
\hline \multicolumn{7}{|l|}{ HOMA-IR } \\
\hline SNP & $0.051(0.016)$ & 0.0017 & $0.048(0.016)$ & 0.0033 & $0.049(0.016)$ & 0.0029 \\
\hline Age & $-0.003(0.001)$ & 0.0012 & $-0.003(0.001)$ & 0.0013 & $-0.003(0.001)$ & 0.0014 \\
\hline Sex & $-0.070(0.025)$ & 0.0050 & $-0.070(0.025)$ & 0.0054 & $-0.070(0.025)$ & 0.0048 \\
\hline $\log _{10}(\mathrm{VFA})$ & $0.502(0.045)$ & $6.4 \times 10^{-27}$ & $0.500(0.045)$ & $1.4 \times 10^{-26}$ & $0.500(0.045)$ & $1.1 \times 10^{-26}$ \\
\hline $\log _{10}$ (adiponectin) & $-0.276(0.057)$ & $1.3 \times 10^{-6}$ & $-0.276(0.057)$ & $1.4 \times 10^{-6}$ & $-0.279(0.057)$ & $1.2 \times 10^{-6}$ \\
\hline \multicolumn{7}{|l|}{ Total cholesterol } \\
\hline SNP & $-0.729(1.826)$ & 0.69 & $-0.934(1.853)$ & 0.61 & $-0.693(1.859)$ & 0.71 \\
\hline Age & $0.383(0.116)$ & 0.0010 & $0.380(0.117)$ & 0.0011 & $0.381(0.116)$ & 0.0011 \\
\hline Sex & $-12.681(2.832)$ & $8.5 \times 10^{-6}$ & $-12.671(2.836)$ & $8.9 \times 10^{-6}$ & $-12.595(2.830)$ & $9.6 \times 10^{-6}$ \\
\hline $\log _{10}(\mathrm{VFA})$ & $26.539(5.141)$ & $3.0 \times 10^{-7}$ & $26.672(5.154)$ & $2.8 \times 10^{-7}$ & $26.589(5.146)$ & $2.9 \times 10^{-7}$ \\
\hline $\log _{10}$ (adiponectin) & $11.912(6.424)$ & 0.064 & $12.197(6.460)$ & 0.059 & $12.168(6.462)$ & 0.060 \\
\hline \multicolumn{7}{|l|}{ Triglycerides } \\
\hline SNP & $0.030(0.011)$ & 0.0054 & $0.025(0.011)$ & 0.024 & $0.026(0.011)$ & 0.020 \\
\hline Age & $0.001(0.001)$ & 0.23 & $0.001(0.001)$ & 0.23 & $0.001(0.001)$ & 0.24 \\
\hline Sex & $0.027(0.017)$ & 0.10 & $0.029(0.017)$ & 0.084 & $0.028(0.017)$ & 0.090 \\
\hline $\log _{10}(\mathrm{VFA})$ & $0.321(0.030)$ & $7.8 \times 10^{-25}$ & $0.320(0.030)$ & $1.6 \times 10^{-24}$ & $0.321(0.030)$ & $1.1 \times 10^{-24}$ \\
\hline $\log _{10}$ (adiponectin) & $-0.258(0.038)$ & $1.8 \times 10^{-11}$ & $-0.253(0.038)$ & $5.6 \times 10^{-11}$ & $-0.254(0.038)$ & $4.7 \times 10^{-11}$ \\
\hline \multicolumn{7}{|l|}{ HDL-cholesterol } \\
\hline SNP & $-2.444(0.682)$ & $3.6 \times 10^{-4}$ & $-2.192(0.693)$ & 0.0016 & $-2.210(0.695)$ & 0.0015 \\
\hline Age & $0.083(0.043)$ & 0.055 & $0.081(0.044)$ & 0.064 & $0.082(0.044)$ & 0.059 \\
\hline Sex & $-0.949(1.058)$ & 0.37 & $-1.011(1.060)$ & 0.34 & $-0.981(1.058)$ & 0.35 \\
\hline $\log _{10}(\mathrm{VFA})$ & $-17.432(1.921)$ & $6.5 \times 10^{-19}$ & $-17.295(1.927)$ & $1.5 \times 10^{-18}$ & $-17.363(1.925)$ & $1.0 \times 10^{-18}$ \\
\hline $\log _{10}$ (adiponectin) & $19.665(2.400)$ & $8.2 \times 10^{-16}$ & $19.617(2.415)$ & $1.4 \times 10^{-15}$ & $19.599(2.417)$ & $1.6 \times 10^{-15}$ \\
\hline \multicolumn{7}{|c|}{ Systolic blood pressure } \\
\hline SNP & $-0.881(0.872)$ & 0.31 & $-0.891(0.886)$ & 0.31 & $-0.948(0.888)$ & 0.29 \\
\hline Age & $0.403(0.056)$ & $1.2 \times 10^{-12}$ & $0.397(0.056)$ & $2.4 \times 10^{-12}$ & $0.402(0.056)$ & $1.4 \times 10^{-12}$ \\
\hline Sex & $-3.085(1.363)$ & 0.024 & $-3.061(1.362)$ & 0.025 & $-3.028(1.362)$ & 0.026 \\
\hline $\log _{10}(\mathrm{VFA})$ & $20.949(2.528)$ & $4.2 \times 10^{-16}$ & $21.143(2.529)$ & $2.4 \times 10^{-16}$ & $21.007(2.531)$ & $3.8 \times 10^{-16}$ \\
\hline $\log _{10}$ (adiponectin) & $1.956(3.053)$ & 0.52 & $2.218(3.064)$ & 0.47 & $2.097(3.070)$ & 0.49 \\
\hline \multicolumn{7}{|c|}{ Diastolic blood pressure } \\
\hline SNP & $0.119(0.563)$ & 0.83 & $0.170(0.572)$ & 0.77 & $0.160(0.574)$ & 0.78 \\
\hline Age & $0.055(0.036)$ & 0.13 & $0.052(0.036)$ & 0.15 & $0.055(0.036)$ & 0.13 \\
\hline Sex & $1.659(0.880)$ & 0.060 & $1.625(0.880)$ & 0.065 & $1.667(0.879)$ & 0.058 \\
\hline $\log _{10}(\mathrm{VFA})$ & $14.946(1.633)$ & $3.5 \times 10^{-19}$ & $15.028(1.635)$ & $2.5 \times 10^{-19}$ & $14.939(1.635)$ & $4.0 \times 10^{-19}$ \\
\hline $\log _{10}$ (adiponectin) & $2.218(1.972)$ & 0.26 & $2.282(1.980)$ & 0.25 & 2.237 (1.983) & 0.26 \\
\hline
\end{tabular}

The effect sizes and $P$-values were derived from linear regression analysis. Fasting plasma glucose, insulin, HOMA-IR, and triglycerides were logtransformed for the analysis. 
would benefit by considering the $\mathrm{CDH} 13$ genotype and measurement of adiponectin levels.

Decreasing adiponectin levels are thought to be associated with the development of insulin resistance and metabolic disorders ${ }^{3,5-8)}$. Adiponectin-inducing alleles of $C D H 13$ SNPs were shown to cause the deterioration of several metabolic syndrome traits. These effects were independent of visceral fat accumulation and adiponectin levels, which affect metabolic syndrome traits, consistent with a previous study by Gao et al. ${ }^{21)}$. The path diagram showed that $C D H 13$ polymorphisms could exacerbate metabolic syndrome, independent of their effect on increasing adiponectin levels. $\mathrm{CDH} 13$ encodes T-cadherin, a receptor for adiponectin $^{18)}$, and $C D H 13$-deficient mice show a phenotype similar to that of adiponectin-deficient mice, without response to adiponectin supplementation ${ }^{27)}$. Since rs3865188 exists $9 \mathrm{~kb}$ upstream of the transcription start site of the $C D H 13$ gene and rs4783244 and rs12051272 in intron 1, these SNPs may affect transcriptional activity. These data suggest that individuals with adiponectin-inducing alleles of $\mathrm{CDH} 13$ have higher adiponectin levels; however, they have increased symptoms of metabolic disorders because of the lower expression of $C D H 13$, an adiponectin receptor.

One of the mechanism through which $\mathrm{CDH} 13$ SNPs may affect the expression of the $\mathrm{CDH} 13$ gene was reported by Putku et al. ${ }^{28)}$, who showed that some of SNPs around the $\mathrm{CpG}$ island within the regulatory region of the $\mathrm{CDH} 13$ gene affect the methylation levels of the gene region. Adiponectin-inducing alleles of SNPs (rs8060301 and rs12444338) were related to hypermethylation within the $\mathrm{CpG}$ island, which would reduce the expression of $\mathrm{CDH} 13$. The three SNPs examined in this study (i.e., rs3865188, rs4783244, and rs12051272) were located near rs8060301 and rs12444338 in linkage disequilibrium (LD, $\mathrm{r}^{2}>0.9$; Supplementary Fig. 1). Therefore, these three SNPs in the CDH13 gene are expected to affect the methylation and expression of $C D H 13$, resulting in the occurrence of metabolic disorders, despite higher adiponectin levels. This phenomenon may be considered adiponectin resistance. T-cadherin has been identified as an adiponectin-binding protein with preference for high-molecular-weight (HMW) adiponectin multimers. However, in this study, we did not measure HMW adiponectin. Because both total and HMW adiponectin are related to metabolic syndrome ${ }^{29,30)}$, further studies are needed to investigate the relationship between CDH13 SNPs and metabolic syndrome including HMW adiponectin levels.

Adiponectin levels are affected by various factors other than age, sex, VFA and CDH13 SNPs. Varia- tions in the adiponectin, C1Q and collagen domain containing $(A D I P O Q)$ gene are also known to determine adiponectin levels and affect metabolic syndrome ${ }^{31)}$. We have recently reported that some $A D I$ $P O Q$ SNPs are associated with adiponectin levels and insulin resistance ${ }^{32)}$. We found that each $A D I P O Q$ (rs10937273 or rs1648707) and CDH13 SNP was independently associated with adiponectin levels. SEM analysis showed that each ADIPOQ SNP affected adiponectin levels, but not risk clustering.

Adiponectin levels have been shown to be increased due to reduced renal function ${ }^{33-35)}$ and decreased by cigarette smoking ${ }^{36)}$. Reduced renal function would decrease the clearance of adiponectin, resulting in an increase in circulating adiponectin levels, even in the healthy subjects. Further studies including renal function and cigarette smoking would support our hypothesis regarding the presence of an adiponectin-resistant state.

\section{Conclusion}

We found that three SNPs (rs3865188, rs4783244, and rs12051272) in the CDH13 gene were associated with increased adiponectin levels; however, these exacerbated metabolic syndrome traits, independent of visceral fat accumulation. These SNPs could affect adiponectin levels and metabolic syndrome traits through the adiponectin-resistant state. Further studies are necessary to clarify the effects of CDH13 SNPs on the adiponectin-resistant state.

\section{Acknowledgments}

We would like to thank Drs. Ryoya Komatsu and Naoto Itoh for collection of samples and data acquisition. This work was supported by Grants-in-Aid from the Ministry of Education, Science, Sports, and Culture of Japan (25461343 to K Hotta, 26460999 to $\mathrm{TK}$, and 26870290 to $\mathrm{AK})$.

\section{COI}

Jun Wada received speaker honoraria from Astellas, Boehringer lngelhelm, Novartis, Novo Nordisk, and Tanabe Mitsubishi, and received grant support from Bayer, Daiichi Sankyo, Kyowa Hakko Kirin, MSD, Novo Nordisk, Otsuka, Torii, Pfizer, Takeda, Taisho Toyama and Tanabe Mitubishi.

Other authors have no potential conflicts of interest associated with this research. 


\section{References}

1) Carr DB, Utzschneider KM, Hull RL, Kodama K, Retzlaff BM, Brunzell JD, Shofer JB, Fish BE, Knopp RH, Kahn SE: Intra-abdominal fat is a major determinant of the National Cholesterol Education Program Adult Treatment Panel III criteria for the metabolic syndrome. Diabetes, 2004; 53: 2087-2094

2) Mastuzawa Y: Metabolic syndrome-definition and diagnostic criteria in Japan. J Atheroscler Thromb, 2005; 12: 301

3) Matsuzawa Y: Therapy insight: adipocytokines in metabolic syndrome and related cardiovascular disease. Nat Clin Pract Cardiovasc Med, 2006; 3: 35-42

4) Maeda K, Okubo K, Shimomura I, Funahashi T, Matsuzawa Y, Matsubara K: cDNA cloning and expression of a novel adipose specific collagen-like factor, apM1 (AdiPose Most abundant Gene transcript 1). Biochem Biophys Res Commun, 1996; 221: 286-289

5) Ouchi N, Kihara S, Arita Y, Maeda K, Kuriyama H, Okamoto Y, Hotta K, Nishida M, Takahashi M, Nakamura T, Yamashita S, Funahashi T, Matsuzawa Y: Novel modulator for endothelial adhesion molecules: adipocyte-derived plasma protein adiponectin. Circulation, 1999; 100(25): 2473-2476

6) Hotta K, Funahashi T, Arita Y, Takahashi M, Matsuda M, Okamoto Y, Iwahashi H, Kuriyama H, Ouchi N, Maeda K, Nishida M, Kihara S, Sakai N, Nakajima T, Hasegawa K, Muraguchi M, Ohmoto Y, Nakamura T, Yamashita S, Hanafusa T, Matsuzawa Y: Plasma concentrations of a novel, adipose-specific protein, adiponectin, in type 2 diabetic patients. Arterioscler Thromb Vasc Biol, 2000; 20: 1595-1599

7) Hotta K, Funahashi T, Bodkin NL, Ortmeyer HK, Arita Y, Hansen BC, Matsuzawa Y: Circulating concentrations of the adipocyte protein adiponectin are decreased in parallel with reduced insulin sensitivity during the progression to type 2 diabetes in rhesus monkeys. Diabetes, 2001; 50: 1126-1133

8) Ryo M, Nakamura T, Kihara S, Kumada M, Shibazaki $S$, Takahashi M, Nagai M, Matsuzawa Y, Funahashi T: Adiponectin as a biomarker of the metabolic syndrome. Circ J, 2004; 68: 975-981

9) Ling H, Waterworth DM, Stirnadel HA, Pollin TI, Barter PJ, Kesäniemi YA, Mahley RW, McPherson R, Waeber G, Bersot TP, Cohen JC, Grundy SM, Mooser VE, Mitchell $\mathrm{BD}$ : Genome-wide linkage and association analyses to identify genes influencing adiponectin levels: the GEMS Study. Obesity, 2009; 17: 737-744

10) Richards JB, Waterworth D, O'Rahilly S, Hivert MF, Loos RJ, Perry JR, Tanaka T, Timpson NJ, Semple RK, Soranzo N, Song K, Rocha N, Grundberg E, Dupuis J, Florez JC, Langenberg C, Prokopenko I, Saxena R, Sladek R, Aulchenko Y, Evans D, Waeber G, Erdmann J, Burnett MS, Sattar N, Devaney J, Willenborg C, Hingorani A, Witteman JC, Vollenweider P, Glaser B, Hengstenberg C, Ferrucci L, Melzer D, Stark K, Deanfield J, Winogradow J, Grassl M, Hall AS, Egan JM, Thompson JR, Ricketts SL, König IR, Reinhard W, Grundy S, Wichmann HE, Barter P, Mahley R, Kesaniemi YA, Rader DJ, Reilly MP, Epstein SE, Stewart AF, Van Duijn CM, Schunkert H,
Burling K, Deloukas P, Pastinen T, Samani NJ, McPherson R, Davey Smith G, Frayling TM, Wareham NJ, Meigs JB, Mooser V, Spector TD; GIANT Consortium: A genome-wide association study reveals variants in ARL15 that influence adiponectin levels. PLoS Genet, 2009; 5: e1000768

11) Heid IM, Henneman P, Hicks A, Coassin S, Winkler T, Aulchenko YS, Fuchsberger C, Song K, Hivert MF, Waterworth DM, Timpson NJ, Richards JB, Perry JR, Tanaka T, Amin N, Kollerits B, Pichler I, Oostra BA, Thorand B, Frants RR, Illig T, Dupuis J, Glaser B, Spector T, Guralnik J, Egan JM, Florez JC, Evans DM, Soranzo N, Bandinelli S, Carlson OD, Frayling TM, Burling K, Smith GD, Mooser V, Ferrucci L, Meigs JB, Vollenweider P, Dijk KW, Pramstaller P, Kronenberg F, van Duijn CM: Clear detection of ADIPOQ locus as the major gene for plasma adiponectin: results of genomewide association analyses including 4659 European individuals. Atherosclerosis, 2010; 208: 412-420

12) Wu Y, Li Y, Lange EM, Croteau-Chonka DC, Kuzawa CW, McDade TW, Qin L, Curocichin G, Borja JB, Lange LA, Adair LS, Mohlke KL: Genome-wide association study for adiponectin levels in Filipino women identifies CDH13 and a novel uncommon haplotype at KNG1ADIPOQ. Hum Mol Genet, 2010; 19: 4955-4964

13) Jee SH, Sull JW, Lee JE, Shin C, Park J, Kimm H, Cho EY, Shin ES, Yun JE, Park JW, Kim SY, Lee SJ, Jee EJ, Baik I, Kao L, Yoon SK, Jang Y, Beaty TH: Adiponectin concentrations: a genome-wide association study. Am J Hum Genet, 2010; 87: 545-552

14) Chung CM, Lin TH, Chen JW, Leu HB, Yang HC, Ho HY, Ting CT, Sheu SH, Tsai WC, Chen JH, Lin SJ, Chen YT, Pan WH: A genome-wide association study reveals a quantitative trait locus of adiponectin on $\mathrm{CDH} 13$ that predicts cardiometabolic outcomes. Diabetes, 2011; 60: 2417-2423

15) Morisaki H, Yamanaka I, Iwai N, Miyamoto Y, Kokubo Y, Okamura T, Okayama A, Morisaki T: CDH13 gene coding T-cadherin influences variations in plasma adiponectin levels in the Japanese population. Hum Mutat, 2012; 33(2): 402-410

16) Dastani Z, Hivert MF, Timpson N, Perry JR, Yuan X, Scott RA, Henneman P, Heid IM, Kizer JR, Lyytikäinen LP, Fuchsberger C, Tanaka T, Morris AP, Small K, Isaacs A, Beekman M, Coassin S, Lohman K, Qi L, Kanoni S, Pankow JS, Uh HW, Wu Y, Bidulescu A, Rasmussen-Torvik LJ, Greenwood CM, Ladouceur M, Grimsby J, Manning AK, Liu CT, Kooner J, Mooser VE, Vollenweider P, Kapur KA, Chambers J, Wareham NJ, Langenberg C, Frants R, Willems-Vandijk K, Oostra BA, Willems SM, Lamina C, Winkler TW, Psaty BM, Tracy RP, Brody J, Chen I, Viikari J, Kähönen M, Pramstaller PP, Evans DM, St Pourcain B, Sattar N, Wood AR, Bandinelli S, Carlson OD, Egan JM, Böhringer S, van Heemst D, Kedenko L, Kristiansson K, Nuotio ML, Loo BM, Harris T, Garcia M, Kanaya A, Haun M, Klopp N, Wichmann HE, Deloukas P, Katsareli E, Couper DJ, Duncan BB, Kloppenburg M, Adair LS, Borja JB; DIAGRAM + Consortium; MAGIC Consortium; GLGC Investigators; MuTHER Consortium, Wilson JG, Musani S, Guo X, 
Johnson T, Semple R, Teslovich TM, Allison MA, Redline S, Buxbaum SG, Mohlke KL, Meulenbelt I, Ballantyne CM, Dedoussis GV, Hu FB, Liu Y, Paulweber B, Spector TD, Slagboom PE, Ferrucci L, Jula A, Perola M, Raitakari O, Florez JC, Salomaa V, Eriksson JG, Frayling TM, Hicks AA, Lehtimäki T, Smith GD, Siscovick DS, Kronenberg F, van Duijn C, Loos RJ, Waterworth DM, Meigs JB, Dupuis J, Richards JB, Voight BF, Scott LJ, Steinthorsdottir V, Dina C, Welch RP, Zeggini E, Huth C, Aulchenko YS, Thorleifsson G, McCulloch LJ, Ferreira T, Grallert H, Amin N, Wu G, Willer CJ, Raychaudhuri S, McCarroll SA, Hofmann OM, Segrè AV, van Hoek M, Navarro P, Ardlie K, Balkau B, Benediktsson R, Bennett AJ, Blagieva R, Boerwinkle E, Bonnycastle LL, Boström $\mathrm{KB}$, Bravenboer B, Bumpstead S, Burtt NP, Charpentier G, Chines PS, Cornelis M, Crawford G, Doney AS, Elliott KS, Elliott AL, Erdos MR, Fox CS, Franklin CS, Ganser M, Gieger C, Grarup N, Green T, Griffin S, Groves CJ, Guiducci C, Hadjadj S, Hassanali N, Herder C, Isomaa B, Jackson AU, Johnson PR, Jørgensen T, Kao WH, Kong A, Kraft P, Kuusisto J, Lauritzen T, Li M, Lieverse A, Lindgren CM, Lyssenko V, Marre M, Meitinger T, Midthjell K, Morken MA, Narisu N, Nilsson P, Owen KR, Payne F, Petersen AK, Platou C, Proença C, Prokopenko I, Rathmann W, Rayner NW, Robertson NR, Rocheleau G, Roden M, Sampson MJ, Saxena R, Shields BM, Shrader P, Sigurdsson G, Sparsø T, Strassburger K, Stringham HM, Sun Q, Swift AJ, Thorand B, Tichet J, Tuomi T, van Dam RM, van Haeften TW, van Herpt T, van Vliet-Ostaptchouk JV, Walters GB, Weedon MN, Wijmenga C, Witteman J, Bergman RN, Cauchi S, Collins FS, Gloyn AL, Gyllensten U, Hansen T, Hide WA, Hitman GA, Hofman A, Hunter DJ, Hveem K, Laakso M, Morris AD, Palmer CN, Rudan I, Sijbrands E, Stein LD, Tuomilehto J, Uitterlinden A, Walker M, Watanabe RM, Abecasis GR, Boehm BO, Campbell H, Daly MJ, Hattersley AT, Pedersen O, Barroso I, Groop L, Sladek R, Thorsteinsdottir U, Wilson JF, Illig T, Froguel P, van Duijn CM, Stefansson K, Altshuler D, Boehnke M, McCarthy MI, Soranzo N, Wheeler E, Glazer NL, Bouatia-Naji N, Mägi R, Randall J, Elliott P, Rybin D, Dehghan A, Hottenga JJ, Song K, Goel A, Lajunen T, Doney A, Cavalcanti-Proença C, Kumari M, Timpson NJ, Zabena C, Ingelsson E, An P, O’Connell J, Luan J, Elliott A, McCarroll SA, Roccasecca RM, Pattou F, Sethupathy P, Ariyurek Y, Barter P, Beilby JP, Ben-Shlomo Y, Bergmann S, Bochud M, Bonnefond A, Borch-Johnsen K, Böttcher Y, Brunner E, Bumpstead SJ, Chen YD, Chines P, Clarke R, Coin LJ, Cooper MN, Crisponi L, Day IN, de Geus EJ, Delplanque J, Fedson AC, Fischer-Rosinsky A, Forouhi NG, Franzosi MG, Galan P, Goodarzi MO, Graessler J, Grundy S, Gwilliam R, Hallmans G, Hammond N, Han X, Hartikainen AL, Hayward C, Heath SC, Hercberg S, Hillman DR, Hingorani AD, Hui J, Hung J, Kaakinen M, Kaprio J, Kesaniemi YA, Kivimaki M, Knight B, Koskinen S, Kovacs P, Kyvik KO, Lathrop GM, Lawlor DA, Le Bacquer O, Lecoeur C, Li Y, Mahley R, Mangino M, Martínez-Larrad MT, McAteer JB, McPherson R, Meisinger C, Melzer D, Meyre D, Mitchell BD, Mukherjee $S$, Naitza $S$, Neville MJ, Orrù M,
Pakyz R, Paolisso G, Pattaro C, Pearson D, Peden JF, Pedersen NL, Pfeiffer AF, Pichler I, Polasek O, Posthuma D, Potter SC, Pouta A, Province MA, Rayner NW, Rice K, Ripatti S, Rivadeneira F, Rolandsson O, Sandbaek A, Sandhu M, Sanna S, Sayer AA, Scheet P, Seedorf U, Sharp SJ, Shields B, Sigurðsson G, Sijbrands EJ, Silveira A, Simpson L, Singleton A, Smith NL, Sovio U, Swift A, Syddall H, Syvänen AC, Tönjes A, Uitterlinden AG, van Dijk KW, Varma D, Visvikis-Siest S, Vitart V, Vogelzangs N, Waeber G, Wagner PJ, Walley A, Ward KL, Watkins H, Wild SH, Willemsen G, Witteman JC, Yarnell JW, Zelenika D, Zethelius B, Zhai G, Zhao JH, Zillikens MC; DIAGRAM Consortium; GIANT Consortium; Global B Pgen Consortium, Borecki IB, Meneton P, Magnusson PK, Nathan DM, Williams GH, Silander K, Bornstein SR, Schwarz P, Spranger J, Karpe F, Shuldiner AR, Cooper C, Serrano-Ríos M, Lind L, Palmer LJ, Hu FB 1st, Franks PW, Ebrahim S, Marmot M, Kao WH, Pramstaller PP, Wright AF, Stumvoll M, Hamsten A; Procardis Consortium, Buchanan TA, Valle TT, Rotter JI, Penninx BW, Boomsma DI, Cao A, Scuteri A, Schlessinger $\mathrm{D}$, Uda M, Ruokonen A, Jarvelin MR, Peltonen L, Mooser V, Sladek R; MAGIC investigators; GLGC Consortium, Musunuru K, Smith AV, Edmondson AC, Stylianou IM, Koseki M, Pirruccello JP, Chasman DI, Johansen CT, Fouchier SW, Peloso GM, Barbalic M, Ricketts SL, Bis JC, Feitosa MF, Orho-Melander M, Melander O, Li X, Li M, Cho YS, Go MJ, Kim YJ, Lee JY, Park T, Kim K, Sim X, Ong RT, Croteau-Chonka DC, Lange LA, Smith JD, Ziegler A, Zhang W, Zee RY, Whitfield JB, Thompson JR, Surakka I, Spector TD, Smit JH, Sinisalo J, Scott J, Saharinen J, Sabatti C, Rose LM, Roberts R, Rieder M, Parker AN, Pare G, O'Donnell CJ, Nieminen MS, Nickerson DA, Montgomery GW, McArdle W, Masson D, Martin NG, Marroni F, Lucas G, Luben R, Lokki ML, Lettre G, Launer LJ, Lakatta EG, Laaksonen R, Kyvik KO, König IR, Khaw KT, Kaplan LM, Johansson $\AA$, Janssens AC, Igl W, Hovingh GK, Hengstenberg C, Havulinna AS, Hastie ND, Harris TB, Haritunians T, Hall AS, Groop LC, Gonzalez E, Freimer NB, Erdmann J, Ejebe KG, Döring A, Dominiczak AF, Demissie S, Deloukas P, de Faire U, Crawford G, Chen YD, Caulfield MJ, Boekholdt SM, Assimes TL, Quertermous T, Seielstad M, Wong TY, Tai ES, Feranil AB, Kuzawa CW, Taylor HA Jr, Gabriel SB, Holm H, Gudnason V, Krauss RM, Ordovas JM, Munroe PB, Kooner JS, Tall AR, Hegele RA, Kastelein JJ, Schadt EE, Strachan DP, Reilly MP, Samani NJ, Schunkert H, Cupples LA, Sandhu MS, Ridker PM, Rader DJ, Kathiresan S: Novel loci for adiponectin levels and their influence on type 2 diabetes and metabolic traits: a multi-ethnic meta-analysis of 45,891 individuals. PLoS Genet, 2012; 8: e1002607

17) Wu Y, Gao H, Li H, Tabara Y, Nakatochi M, Chiu YF, Park EJ, Wen W, Adair LS, Borja JB, Cai Q, Chang YC, Chen P, Croteau-Chonka DC, Fogarty MP, Gan W, He CT, Hsiung CA, Hwu CM, Ichihara S, Igase M, Jo J, Kato N, Kawamoto R, Kuzawa CW, Lee JJ, Liu J, Lu L, McDade TW, Osawa H, Sheu WH, Teo Y, Vadlamudi S, Van Dam RM, Wang Y, Xiang YB, Yamamoto K, Ye X, Young TL, Zheng W, Zhu J, Shu XO, Shin C, Jee SH, 
Chuang LM, Miki T, Yokota M, Lin X, Mohlke KL, Tai ES: A meta-analysis of genome-wide association studies for adiponectin levels in East Asians identifies a novel locus near WDR11-FGFR2. Hum Mol Genet, 2014; 23: 1108-1119

18) Hug C, Wang J, Ahmad NS, Bogan JS, Tsao TS, Lodish HF: T-cadherin is a receptor for hexameric and highmolecular-weight forms of Acrp30/adiponectin. Proc Natl Acad Sci U S A, 2004; 101: 10308-10313

19) Park KG, Park KS, Kim MJ, Kim HS, Suh YS, Ahn JD, Park KK, Chang YC, Lee IK: Relationship between serum adiponectin and leptin concentrations and body fat distribution. Diabetes Res Clin Pract, 2004; 63: 135-142

20) Fava C, Danese E, Montagnana M, Sjögren M, Almgren P, Guidi GC, Hedblad B, Engström G, Lechi A, Minuz P, Melander O: A variant upstream of the $\mathrm{CDH} 13$ adiponectin receptor gene and metabolic syndrome in Swedes. Am J Cardiol, 2011; 108: 1432-1437

21) Gao H, Kim YM, Chen P, Igase M, Kawamoto R, Kim MK, Kohara K, Lee J, Miki T, Ong RT, Onuma H, Osawa H, Sim X, Teo YY, Tabara Y, Tai ES, van Dam RM: Genetic variation in CDH13 is associated with lower plasma adiponectin levels but greater adiponectin sensitivity in East Asian populations. Diabetes, 2013; 62: 4277-4283

22) Yoshizumi T, Nakamura T, Yamane $M$, Islam $A H$, Menju M, Yamasaki K, Arai T, Kotani K, Funahashi T, Yamashita S, Matsuzawa Y: Abdominal fat: standardized technique for measurement at CT. Radiology, 1999; 211: 283-286

23) Ohnishi Y, Tanaka T, Ozaki K, Yamada R, Suzuki H, Nakamura Y: A high-throughput SNP typing system for genome-wide association studies. J Hum Genet, 2001; 46: $471-477$

24) Barrett JC, Fry B, Maller J, Daly MJ: Haploview: analysis and visualization of LD and haplotype maps. Bioinformatics, 2005; 21: 263-265

25) Takahara M, Katakami N, Kaneto H, Noguchi M, Shimomura I: Contribution of visceral fat accumulation and adiponectin to the clustering of metabolic abnormalities in a Japanese population. J Atheroscler Thromb, 2014; 21: 543-553

26) Rosseel Y: lavaan: An R Package for Structural Equation. Modeling J Statistical Software, 2012; 48: 1-36

27) Denzel MS, Scimia MC, Zumstein PM, Walsh K, RuizLozano P, Ranscht B: T-cadherin is critical for adiponectin-mediated cardioprotection in mice. J Clin Invest, 2010; 120: 4342-4352

28) Putku M, Kals M, Inno R, Kasela S, Org E, Kožich V,
Milani L, Laan M: CDH13 promoter SNPs with pleiotropic effect on cardiometabolic parameters represent methylation QTLs. Hum Genet, 2015; 134: 291-303

29) Miyazaki T, Hiki M, Shimada K, Kume A, Kiyanagi T, Sumiyoshi K, Ohmura H, Daida H: The high molecular weight adiponectin level is associated with the atherogenic lipoprotein profiles in healthy Japanese males. J Atheroscler Thromb, 2014; 21: 672-679

30) Matsuda M, Tamura R, Kishida N, Segawa T, Kanno K, Nishimoto O, Nakamoto K, Nishiyama H, Kawamoto T: Predictive value of adiponectin in patients with multivessel coronary atherosclerosis detected on computed tomography angiography. J Atheroscler Thromb, 2013; 20: 767776

31) Kawai T, Ohishi M, Takeya Y, Onishi M, Ito N, Yamamoto K, Oguro R, Kamide K, Rakugi H: Adiponectin single nucleotide polymorphism is a genetic risk factor for stroke through high pulse wave pressure: a cohort study. J Atheroscler Thromb, 2013; 20: 152-160

32) Kitamoto A, Kitamoto T, So R, Matsuo T, Nakata $Y$, Hyogo H, Ochi H, Nakamura T, Kamohara S, Miyatake N, Kotani K, Mineo I, Wada J, Ogawa Y, Yoneda M, Nakajima A, Funahashi T, Miyazaki S, Tokunaga K, Masuzaki H, Ueno T, Chayama K, Hamaguchi K, Yamada K, Hanafusa T, Oikawa S, Sakata T, Tanaka K, Matsuzawa Y, Hotta K: ADIPOQ polymorphisms are associated with insulin resistance in Japanese women. Endocr J, 2015; 62: 513-521

33) Tsuboi A, Watanabe M, Kazumi T, Fukuo K: Anemia and reduced renal function are independent predictors of elevated serum adiponectin in elderly women. J Atheroscler Thromb, 2013; 20: 568-574

34) Takahara M, Katakami N, Kishida K, Kaneto H, Funahashi T, Shimomura I, Matsunaga S, Kubo S, Fukamizu H, Otsuka A, Ichihara K, Nakamura T: Circulating adiponectin levels and their associated factors in young lean healthy Japanese women. J Atheroscler Thromb, 2013; 20: 57-64

35) Tsuboi A, Watanabe M, Kazumi T, Fukuo K: Association of low serum iron levels with low-grade inflammation and hyperadiponectinemia in community-living elderly women. J Atheroscler Thromb, 2013; 20: 670-677

36) Al-Attas OS, Hussain T, Al-Daghri NM, De Rosas E, Kazmi U, Vinodson B: The relationship between a Mediterranean diet and circulating adiponectin levels is influenced by cigarette smoking. J Atheroscler Thromb, 2013; 20: $313-320$ 

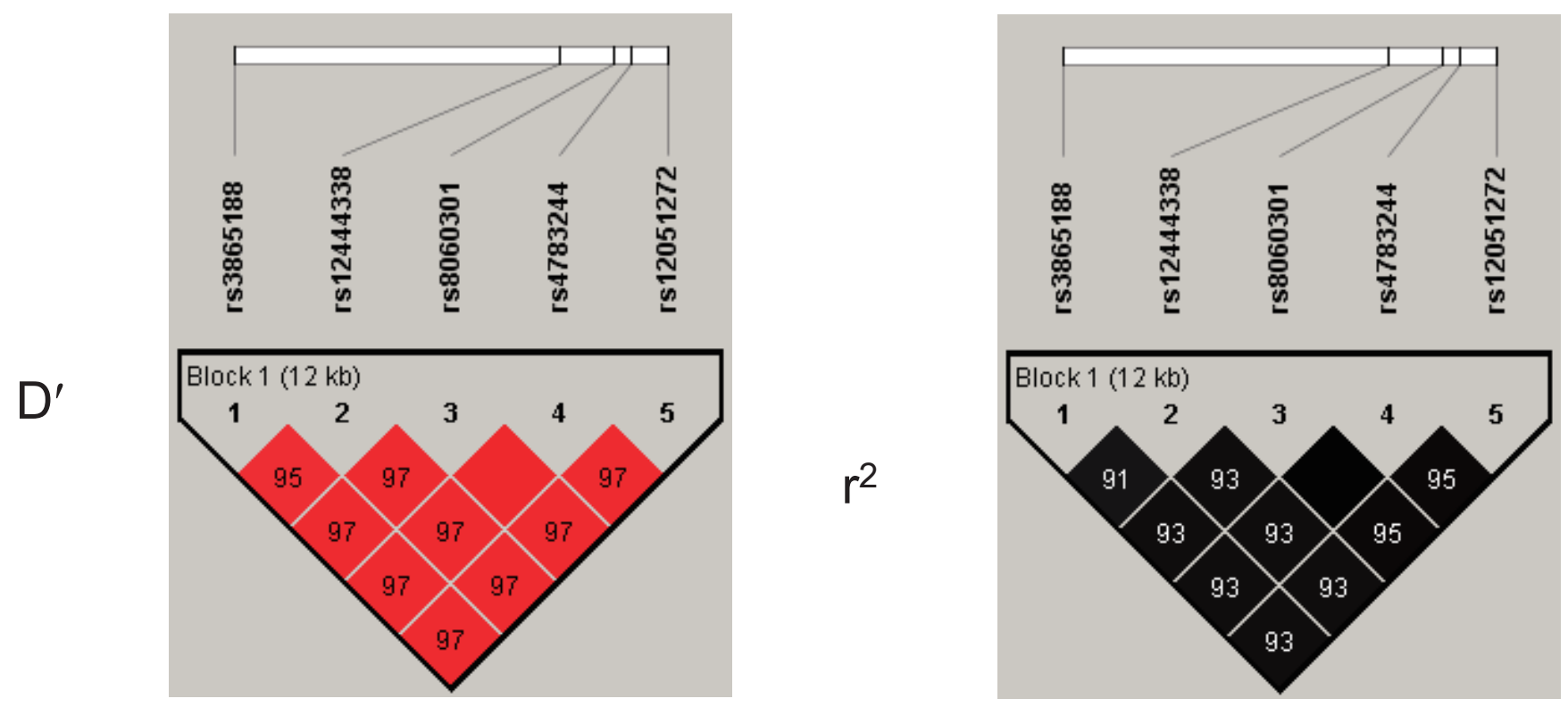

Supplementary Fig. 1. LD map of SNPs in CDH13 